\title{
Relationship among oral habits, orofacial function and oral health- related quality of life in children
}

\section{Marina Severi Leme Taís de Souza Barbosa Maria Beatriz Duarte Gavião}

Department of Pediatric Dentistry, Piracicaba Dental School, Univ of Campinas - UNICAMP, Piracicaba, SP, Brazil.
Declaration of Interests: The authors certify that they have no commercial or associative interest that represents a conflict of interest in connection with the manuscript.

\section{Corresponding Author:} Maria Beatriz Duarte Gavião

E-mail:mbgaviao@fop.unicamp.br

Submitted: Oct 18, 2012 Accepted for publication: Feb 06, 2013 Last revision: Feb 25, 2013

\begin{abstract}
The objective was to evaluate the relationship among oral habits, oral function and oral health-related quality of life (OHRQoL) in children. Three hundred and twenty-eight subjects (8-14 years old) were assessed for orofacial function using the Brazilian version of the Nordic Orofacial Test-Screening (NOT-S). OHRQoL was assessed using the Child Perceptions Questionnaires (Brazilian versions) for the 8-10 $\left(\mathrm{CPQ}_{8-10}\right)$ and 11-14 $\left(\mathrm{CPQ}_{11-14}\right)$ year age groups. The subjects were distributed into a Habit group and a Habit-free group according to domain III (Habits) of the NOT-S. Oral habits were present in $71.3 \%$ of the sample $(p=.0001)$, with a higher prevalence in females $(62.8 \%, p=.001)$. The NOT-S, $\mathrm{CPQ}_{8-10}$ and $\mathrm{CPQ}_{11-14}$ scores were higher in the Habit group $(P=.0001, P=.009$ and $p=.001$, respectively). Domain I (Sensory Function) was significantly more affected in Habit group subjects $(p=.001)$. The NOT-S scores were positively correlated with the $\mathrm{CPQ}_{8-10}$ and $\mathrm{CPQ}_{11-14}$ scores only in the Habit group $(\mathrm{r}=.32, p=.0003$ and $\mathrm{r}=.30$, $p=.001$, respectively). These results indicate that oral habits can impact OHRQoL. Moreover, orofacial dysfunctions were associated with worse OHRQoL in subjects with oral habits.
\end{abstract}

Descriptors: Disability Evaluation; Habits; Oral Health; Quality of Life.

\section{Introduction}

The World Health Organization (WHO) defines health as a state of complete physical, social and mental well-being and defines quality of life (QoL) as the individual's perception of their position in life in the context of the culture and value system where they live and in relation to their goals, expectations, standards, and concerns. ${ }^{1}$ Thus, health influences QoL. Oral health is an important part of general health and well-being and is more important for the general health of those with oral or craniofacial problems. ${ }^{2}$ Over the past two decades, traditional methods of measuring oral health, which mainly use clinical dental indices and focus only on the absence or presence of oral diseases, ${ }^{3}$ have been substituted for a multidimensional concept that includes the psychosocial aspects of oral health ${ }^{4}$ and their influence on oral health-related quality of life (OHRQoL).

Instruments for OHRQoL measurement have been developed to assess the physical and psycho-social impact of oral health, to quantify the 
extent to which dental and oral disorders interfere with daily life and well-being ${ }^{5}$ and to assess both the needs for and outcomes of clinical and psychosocial interventions from the perspective of the individual concerned. ${ }^{6}$ These instruments have the potential to improve the quality of clinical and psychosocial care. $^{7}$

Orofacial function includes vital actions (e.g., breathing, chewing and swallowing) and muscle posture (e.g., mouth and tongue posture $)^{8}$ and forms the basis for social interaction in terms of speech, emotional communication, facial expression and appearance. ${ }^{9}$ Orofacial dysfunctions can compromise well-being and OHRQoL from childhood. ${ }^{10}$ There is evidence of the importance of comprehending the individual and ambient factors that influence the relationship between orofacial function and health/ QoL. Children are affected by numerous oral and orofacial disorders (e.g., dental caries, malocclusion, cleft lip and palate), all of which have the potential to compromise functioning, well-being and OHRQoL. ${ }^{10}$

Orofacial dysfunctions also include the presence of oral habits, ${ }^{10}$ which are the main functional factors that influence the development of malocclusion. Sucking habits or nail biting, mouth breathing and alterations of swallowing are the most common. ${ }^{11}$

The present study aimed to evaluate the influence of oral habits on orofacial functions and OHRQoL in children. An additional aim was to verify if there were associations between orofacial dysfunctions and OHRQoL in groups with and without oral habits.

\section{Methodology Sample}

This project was approved by the Ethics Committee in Research of Piracicaba Dental School, University of Campinas (CEP-FOP; protocol number 009/2008). Four public schools in Piracicaba, SP, Brazil, were selected by lottery. A meeting was scheduled with parents/guardians to explain the study aims and methods to be applied and to distribute consent forms. During this period, a total of 550 consent forms were distributed, and 333 were returned with the respective parent/guardian autho- rizations. The exclusion criteria were as follows:

- presence of systemic and/or mental developmental disorders as reported by teachers and coordinators of school and

- non-collaboration with the examinations and fulfillment of the OHRQoL questionnaire, which is self-applied (Child Perceptions Questionnaires, CPQ). ${ }^{12,13}$

The final sample was composed of 328 children, 8 to 14 years old (mean age: $1.5 \pm 1.7$ years), including 197 girls (60.1\%) and 131 boys (39.9\%). The age range was established according to the CPQ ages, 8-10 and 11-14 years old, as detailed below. Five children out of 333 were excluded because they did not complete the CPQ.

The assessments were carried out on two week days during the 2009 academic year. During the first assessment, orofacial function was evaluated, and during the second assessment, the questionnaires were applied.

\section{Orofacial functions}

Orofacial functions were evaluated using the Nordic Orofacial Test-Screening (NOT-S) protocol, ${ }^{11}$ translated and culturally adapted to Brazilian Portuguese. ${ }^{14}$ This protocol consists of a structured interview and a clinical examination. Each part has six domains. In the interview, the following functions are assessed:

(I) sensory function,

(II) breathing,

(III) habits,

(IV) chewing and swallowing,

(V) drooling and

(IV) dryness of the mouth.

In the examination, the following functions are assessed:

(1) face at rest,

(2) nose breathing,

(3) facial expression,

(4) masticatory muscle and jaw function,

(5) oral motor function and

(6) speech. 
Each domain contains one to five items, reflecting the complexity of the specific function.

NOT-S was applied individually (MSL). The NOT-S interview was performed by asking questions on the screening form, for example, in item I "Sensory function," one question is "Does brushing your teeth elicit a gag reflex?" To assess orofacial dysfunctions in the clinical examination, the subjects were requested to conduct tasks related to items 1-6 and an illustrated manual, which contained pictures of the tasks, was used. An answer of "yes" or a task that met the criteria for impaired function resulted in a score of 1 , indicating a dysfunction in the scored domain. An answer of "no" or a task that did not meet the criteria resulted in a score of zero. The total score was the sum of the score for each domain, ranging from 0 to 12 . For more details, please consult http://mun-h-center.se/ en/Mun-H-Center/Mun-H-Center-E/NOT-S/.

The subjects were included in the Habit group if they answered yes to at least one of the three questions in domain III of the NOT-S and in the Habitfree group if they answered no to all questions in this domain. The types of oral habits asked about and recorded were:

- nail biting,

- lip biting or sucking,

- thumb or finger sucking,

- pacifier sucking,

- bottle sucking,

- cheek biting,

- tooth grinding,

- tongue biting and

- pencil and pen biting.

\section{Oral health-related quality of life (OHRQOL)}

The OHRQoL was evaluated using the CPQ, ${ }^{12,13}$ validated for Brazilian children, ${ }^{15}$ which takes into account the cognitive abilities and lifestyles in an age range from 8 to 10 years $\left(\mathrm{CPQ}_{8-10}\right)$ and from 11 to 14 years $\left(\mathrm{CPQ}_{11-14}\right)$. The instrument assesses the perceptions of the impact of oral disorders on physical and psychosocial functioning. The CPQ items are distributed into four domains:

- oral symptoms,
- functional limitations (e.g., difficulties with chewing),

- emotional well-being and

- social well-being.

A Likert-type scale was used with the response options of

- never $=0$,

- once or twice $=1$,

- sometimes $=2$,

- often $=3$, and

- very often $=4$.

A high score indicated more negative impacts on OHRQoL. The $\mathrm{CPQ}_{8-10}$ is composed of 29 questions and the $\mathrm{CPQ}_{11-14}$ of 41 . The minimum possible score is zero, and the maximum possible score is $\mathbf{1 1 6}$ for the $\mathrm{CPQ}_{8-10}$ and 164 for the $\mathrm{CPQ}_{11-14}$.

All children completed the age-specific CPQ by themselves. First, the researchers read the instructions, and the children were requested to inquire if they had any questions.

\section{Statistical analysis}

Data analysis was performed using SPSS 9.0 software (SPSS, Chicago, USA) with a 5\% significance level. Normality was assessed using the KolmogorovSmirnov test. Because score distributions were asymmetrical, non-parametrical tests were used. Descriptive statistics were followed by bivariate analyses, chi-squared and Fisher's exact tests for a comparison of proportions (number of individuals and gender) and the Mann-Whitney test for a comparison of the means of the continuous variables (age, NOT-S and CPQ scores). Differences in the means of the NOT-S and CPQ scores according to different types of oral habits were evaluated using the Kruskal-Wallis test. Spearman's correlation test was used to evaluate the correlation between the NOT-S and both CPQ scores for each clinical group (Habit and Habit free).

\section{Results}

The sample characteristics are shown in Table 1. The majority of the subjects $(71.3 \%, p<.0001)$ presented at least one oral habit and were included in the Habit group. Females were more frequent in the 
Table 1 - Descriptive data of the analyzed variables according to the clinical groups.

\begin{tabular}{|c|c|c|c|c|}
\hline & & Habit & Habit free & $p^{c}$ \\
\hline \multicolumn{2}{|l|}{ Number $(\%)^{a}$} & 234 (71.3) & 94 (28.7) & $<.001$ \\
\hline \multicolumn{2}{|l|}{ Mean age $(S D)^{b}$} & $1.6(1.7)$ & $1.0(1.7)$ & .19 \\
\hline \multicolumn{5}{|l|}{ Gender [n (\%)] } \\
\hline \multicolumn{2}{|l|}{ Boy } & 87 (37.2) & $44(46.8)$ & - \\
\hline \multirow{2}{*}{\multicolumn{2}{|c|}{ Girl }} & $147(62.8)$ & $50(53.2)$ & - \\
\hline & & $p^{d}=.001$ & $p^{d}=.70$ & \\
\hline \multicolumn{5}{|l|}{ NOT-S scores } \\
\hline \multicolumn{2}{|l|}{ Mean $(S D)^{b}$} & $3.0(1.4)$ & $2.0(1.3)$ & $<.001$ \\
\hline \multicolumn{2}{|l|}{ Median (IQR) } & $3(2)$ & $2(2)$ & \\
\hline \multicolumn{5}{|c|}{ Mean $C P Q$ scores $(S D)^{b}$} \\
\hline \multirow{2}{*}{$8-10(n=173)$} & Mean (SD) & $14.6 \pm 12.9$ & $1.1 \pm 11.1$ & .009 \\
\hline & Median (IQR) & $12(13)$ & $8(10)$ & \\
\hline \multirow{2}{*}{$11-14(n=155)$} & Mean (SD) & $24.6 \pm 17.8$ & $15.7 \pm 13.7$ & .001 \\
\hline & Median (IQR) & $20(23)$ & $9(12)$ & \\
\hline
\end{tabular}

$\mathrm{SD}$, standard deviation; IQR, interquartile range; ${ }^{a} \mathrm{chi}$-square test; ${ }^{\mathrm{b}}$ Mann-Whitney test; ${ }^{\mathrm{c}} \mathrm{p}$-value (differences between columns); ${ }^{d} p$-value (differences between lines).
Table 2 - NOT-S and CPQ scores according to the oral habit type.

\begin{tabular}{|c|c|c|c|c|}
\hline Type of oral habit & & NOT-S scores & $C P Q_{8-10}$ & $C P Q_{11-14}$ \\
\hline \multirow{2}{*}{$\begin{array}{l}\text { Thumb or finger } \\
\text { sucking }(n=21)\end{array}$} & Mean (SD) & $3.2 \pm 1.3$ & $14.8 \pm 13.4$ & $36.7 \pm 18.6$ \\
\hline & Median (IQR) & $3(2)$ & $9(12.5)$ & $36.5(11.75)$ \\
\hline \multirow{2}{*}{ Pacifier sucking $(n=2)$} & Mean (SD) & $4.0 \pm 4.2$ & $15.0 \pm 14.1$ & - \\
\hline & Median (IQR) & $4(3)$ & $15(10)$ & \\
\hline \multirow{2}{*}{ Bottle sucking $(n=2)$} & Mean (SD) & $2.0 \pm 1.4$ & - & - \\
\hline & Median (IQR) & $2(1)$ & & \\
\hline \multirow{2}{*}{ Nail biting $(n=141)$} & Mean (SD) & $3.0 \pm 1.3$ & $15.8 \pm 12.4$ & $24.8 \pm 19.1$ \\
\hline & Median (IQR) & $3(2)$ & $13(12)$ & $19(24.5)$ \\
\hline \multirow{2}{*}{$\begin{array}{l}\text { Lip biting or sucking } \\
(\mathrm{n}=89)\end{array}$} & Mean (SD) & $3.0 \pm 1.5$ & $12.1 \pm 1.9$ & $24.0 \pm 19.8$ \\
\hline & Median (IQR) & $3(2)$ & $9(11.25)$ & $19(22)$ \\
\hline \multirow{2}{*}{ Cheek biting $(n=52)$} & Mean (SD) & $3.3 \pm 1.5$ & $14.8 \pm 15.9$ & $29.3 \pm 16.2$ \\
\hline & Median (IQR) & $3(2)$ & $6(14.25)$ & $27(25)$ \\
\hline \multirow{2}{*}{ Grinding $(n=49)$} & Mean (SD) & $3.5 \pm 1.5$ & $17.6 \pm 16.5$ & $25.6 \pm 2.0$ \\
\hline & Median (IQR) & $3(1)$ & $9(21.25)$ & $21(23)$ \\
\hline \multirow{2}{*}{ Tongue biting $(\mathrm{n}=20)$} & Mean (SD) & $3.6 \pm 1.6$ & $17.2 \pm 13.9$ & $28.7 \pm 18.8$ \\
\hline & Median (IQR) & $3(5)$ & $18(15)$ & $27(22)$ \\
\hline \multirow{2}{*}{$\begin{array}{l}\text { Pencil or pen biting } \\
(n=4)\end{array}$} & Mean (SD) & $3.0 \pm 2.0$ & - & $27.3 \pm 17.9$ \\
\hline & Median (IQR) & $2(1)$ & & $27(27.75)$ \\
\hline \multicolumn{2}{|l|}{$p$-value ${ }^{a}$} & .29 & .56 & .22 \\
\hline
\end{tabular}

a Kruskal-Wallis test (differences between lines). 
Table 3 - Distribution of the sample [n (\%)] for each NOT-S domain.

\begin{tabular}{|c|c|c|c|c|c|}
\hline \multirow[t]{2}{*}{ NOT-S domains } & \multicolumn{2}{|c|}{$\begin{array}{c}\text { Habit } \\
(n=234)\end{array}$} & \multicolumn{2}{|c|}{$\begin{array}{l}\text { Habit free } \\
(\mathrm{n}=94)\end{array}$} & \multirow[t]{2}{*}{$p$-value } \\
\hline & $\mathrm{N}$ & $\%$ & $\mathrm{~N}$ & $\%$ & \\
\hline \multicolumn{6}{|l|}{ Interview } \\
\hline (I) Sensory function & 41 & 18.0 & 2 & 2.1 & .001 \\
\hline (II) Breathing & 22 & 9.4 & 9 & 9.6 & .87 \\
\hline (III) Habits & 234 & 10.0 & 0 & 0 & - \\
\hline $\begin{array}{l}\text { (IV) Chewing and } \\
\text { swallowing }\end{array}$ & 125 & 53.0 & 41 & 44.0 & .41 \\
\hline (V) Drooling & 29 & 12.0 & 13 & 14.0 & .90 \\
\hline (VI) Dry mouth & 64 & 27.0 & 16 & 17.0 & .15 \\
\hline \multicolumn{6}{|l|}{ Examination } \\
\hline (1) Face at rest & 67 & 29.0 & 31 & 33.0 & .66 \\
\hline (2) Nose breathing & 9 & 3.8 & 5 & 5.3 & .55 \\
\hline (3) Facial expression & 64 & 27.0 & 28 & 3.0 & .84 \\
\hline $\begin{array}{c}\text { (4) Masticatory muscle } \\
\text { and jaw function }\end{array}$ & 25 & 11.0 & 14 & 15.0 & .45 \\
\hline $\begin{array}{l}\text { (5) Oral motor } \\
\text { function }\end{array}$ & 5 & 2.1 & 6 & 6.4 & .09 \\
\hline (6) Speech & 14 & 6.0 & 6 & 6.4 & .10 \\
\hline
\end{tabular}

${ }^{\circ}$ Comparisons with the Habit-free group (chi-square and Fisher's exact tests, where appropriate).

Habit group $(62.8 \%, p=.001)$. Children with oral habits had, on average, higher mean NOT-S (3.0 versus $2.0 ; \mathrm{p}<.001), \mathrm{CPQ}_{8-10}(14.6$ versus $1.1 ; p=.009)$ and $\mathrm{CPQ}_{11-14}$ scores $(24.6$ versus $15.7 ; p=.001)$ than subjects in the Habit-free group. When considering the types of oral habits, there were no differences in the means of the NOT-S and both CPQ scores $(\mathrm{p}>.05)$ (Table 2).

Table 3 shows the sample distribution for each domain of the NOT-S. Children with oral habits had higher impairment of sensory function (Domain I) than the children in the Habit-free group $(p=.001)$.

For the Habit group, the NOT-S scores were positively correlated with the $\mathrm{CPQ}_{8-10}$ and $\mathrm{CPQ}_{11-14}$ scores. There were no correlations between the NOT-S and both CPQ scores in the Habit-free group (Table 4).

\section{Discussion}

The NOT-S and CPQ were chosen because they
Table 4 - Spearman's correlation between NOT-S and $C P Q$ scores.

\begin{tabular}{l|c|c|c|c}
\hline & \multicolumn{2}{|c|}{ Habit $(\mathrm{n}=234)$} & \multicolumn{2}{c}{ Habit free $(\mathrm{n}=94)$} \\
\cline { 2 - 5 } & $\mathrm{R}$ & $\mathrm{p}$-value & $\mathrm{R}$ & $\mathrm{p}$-value \\
\hline NOT-S and $\mathrm{CPQ}_{8-10}$ & .32 & $<.001$ & .16 & .24 \\
\hline NOT-S and $\mathrm{CPQ}_{11-14}$ & .30 & .001 & .12 & .45 \\
\hline
\end{tabular}

are validated protocols available in many languages, including Brazilian Portuguese, ${ }^{12,15}$ making possible the comparison of international data, assessing orofacial functions and OHRQoL.

A wide range of frequencies of oral habits has been reported, from $9.9 \%$ to $34.1 \%$ on international studies, ${ }^{16,17}$ and from $70 \%$ to $83.1 \%$ in Brazilian studies, ${ }^{18,19}$ corroborating our results. The most prevalent habit was nail biting. Pacifier and finger sucking were less prevalent, which agrees with the findings of Thomaz et al. ${ }^{20}$ These results can be justified by the age sample because it has been observed that sucking habits during early ages may further change to nail biting or another oral habit ${ }^{20}$ if psychological and/or emotional factors are involved. ${ }^{21}$

A higher prevalence of oral habits was found in girls than in boys. To date, there is no consensus regarding the prevalence of oral habits and their association with gender. The lack of consensus might be explained by the different dentition stages of the evaluated populations. ${ }^{11,19,22}$

The Habit group presented higher $\mathrm{CPQ}_{8-10}$ and $\mathrm{CPQ}_{11-14}$ scores than the Habit-free group, indicating that the consequences of oral habits on oral health could be considered as influential factors on quality of life, corroborating the findings of Sardenberg et al. ${ }^{23} \mathrm{~A}$ possible explanation is that the maintenance of oral habits can be the etiological factor of malocclusion, and it is been suggested that malocclusion has a high impact on OHRQoL. ${ }^{2,23}$ Another possibility is that individuals with a gag reflex felt that their oral condition had an impact on overall QoL. ${ }^{24}$ This observation could justify the higher CPQ scores for the Habit group, which presented this sensory function on the NOT-S evaluation. Moreover, girls reported a higher impact on OHRQoL than boys, ${ }^{15,25}$ and the Habit group was $62.8 \%$ girls. Teeth appearance was important for girls and had a 
high impact on their appearance satisfaction..$^{25}$

Subjects with oral habits also presented higher NOT-S scores than their counterparts. When considering the sample distribution according to each domain of NOT-S, there were significant differences between the Habit and Habit-free groups, with the former presenting higher impairment on sensory function (Domain I) than the latter. The sensory function in the NOT-S is evaluated by asking the individual if toothbrushing elicits a gag reflex and if having too much food in the mouth makes it difficult to chew. The sensory function in children in the Habit group was considered impaired due to the presence of gag reflexes when tooth brushing, indicating the influence of oral habits in this NOT-S domain. Deleterious oral habits may be related to emotional and/or psychological factors, ${ }^{21}$ particularly nail biting, ${ }^{26}$ which was the most prevalent habit in the present study. In addition, the gag reflex can be an expression of emotional distress, ${ }^{27}$ and patients with a gag reflex have been found to be dentally anxious. ${ }^{28}$ In this context, the observed relationship between the oral habits and gag reflex could be a manifestation of stress related to the oral cavity, although verifying this possibility requires further research. Nevertheless, a low number of children demonstrated impairment in some domains of clinical examination that can be considered limitations, such as nose breathing, oral motor function and speech.

Unexpectedly, the breathing domain results in the NOT-S interview were similar in the two groups, which is justified by the healthy sample, i.e., the participants were chosen randomly from a population, differing from the choice by Bakke et al. ${ }^{9}$ Further-

\section{References}

1. World Health Organization. Health Promotion Glossary [Internet]. Geneva: World Health Organization; 1986. [cited 2012 Oct 15]. Available from: http://whqlibdoc.who.int/ hq/1998/WHO_HPR_HEP_98.1.pdf.

2. Petersen PE. The World Oral Health report 2003: continuous improvement of oral health in the 21st century - the approach of the WHO Global Oral Health Programme. Community Dent Oral Epidemiol 2003 Dec;31Suppl.1:3-24. more, the most prevalent oral habits could not influence morphological characteristics related to oral breathing onset. Chewing and swallowing were also the same for both groups, as observed in the NOT$S$ interview, indicating that the oral habits did not influence the respective functions. Both interview domains results were in line with the NOT-S clinical examination, indicating consistent results.

The NOT-S, $\mathrm{CPQ}_{8-10}$ and $\mathrm{CPQ}_{11-14}$ scores presented significant correlations in the Habit group, indicating the influence of orofacial dysfunctions on OHRQoL. This finding is in line with the literature because oral health is related to well-being and QoL, as measured along functional, psychosocial, and economic dimensions. ${ }^{9}$ Child OHRQoL can be influenced by dental caries, fluorosis, malocclusion, gum problems, cleft lip and palate and/or craniofacial anomalies. ${ }^{15}$ Moreover, oral and craniofacial diseases or disabilities contribute to the compromise of oral sensorimotor functions ${ }^{3}$ and, consequently, negatively influence the OHRQoL.

\section{Conclusions}

Children and adolescents of the Habit group presented more orofacial dysfunction and higher impacts on OHRQoL than the habit-free group. Moreover, the presence of orofacial dysfunctions was associated with worse OHRQoL in subjects with oral habits.

\section{Acknowledgements}

The authors gratefully acknowledge the financial support provided by the State of São Paulo Research Foundation (FAPESP, SP, Brazil, n. 2007/06863-0), the volunteers and their parents.

3. Robinson PG, Gibson B, Khan F, Birnbaum W. Validity of two oral health-related quality of life measures. Community Dent Oral Epidemiol. 2003 Apr;31(2):90-9.

4. Gift HC, Atchison KA, Dayton CM. Conceptualizing oral health and oral health-related quality of life. Soc Sci Med. 1997 Mar;44(5):601-8.

5. Barbosa TS, Gavião MBD. Oral health-related quality of life in children: Part II. Effects of clinical oral health status. A systematic review. Int J Dent Hyg. 2008 May;6(2):100-7. 
6. Locker D, Jokovic A, Tompson B. Health-related quality of life of children aged 11 to 14 years with orofacial conditions. Cleft Palate Craniofac J. 2005 May;42(3):260-6.

7. Marcusson A, Akerlind I, Paulin G. Quality of life in adults with repaired cleft lip and palate. Cleft Palate Craniofac J. $2001 \mathrm{Jul} ; 38(4): 379-85$.

8. Grabowski R, Kundt G, Stahl F. Interrelation between occlusal findings and orofacial myofunctional status in primary and mixed dentition - part III: interrelation between malocclusions and orofacial dysfunctions. J Orofac Orthop. 2007 Nov;68(6):462-76.

9. Bakke M, Bergendal B, McAllister A, Sjogreen L, Asten P. Development and evaluation of a comprehensive screening for orofacial dysfunction. Swed Dent J. 2007;31(2):75-84.

10. Department of Health and Human Services (U.S.). Oral health in America: a report of the surgeon general. Rockville (MD): Department of Health and Human Services (U.S.), National Institute of Dental and Craniofacial Research, National Institutes of Health; 2000. 322 p.

11. Stahl F, Grabowski R, Gaebel M, Kundt G. Relationship between occlusal findings and orofacial myofunctional status in primary and mixed dentition - Part II: prevalence of orofacial dysfunctions. J Orofac Orthop. 2007 Mar;68(2):74-9.

12. Jokovic A, Locker D, Stephens M, Kenny D, Tompson B, Guyatt G. Validity and reliability of a questionnaire for measuring child oral-health-related quality of life. J Dent Res. 2002 Jul;81(7):459-63.

13. Jokovic A, Locker D, Tompson B, Guyatt G. Questionnaire for measuring oral health-related quality of life in eight- to tenyear-old children. Pediatr Dent. 2004 Nov-Dec;26(6):512-8.

14. Leme MS, Barbosa TS, Gavião MBD. Brazilian version of the Nordic Orofacial Test - Screening (NOT-S) for evaluation of orofacial dysfunctions. Pesqui Bras Odontopediatria Clin Integr. 2012 Apr-May;11(2):281-9. Portuguese.

15. Barbosa TS, Tureli MCM, Gavião MBD. Validity and reliability of the child perceptions questionnaires applied in Brazilian children. BMC Oral Health. 2009 May;18:9-13. doi: 10.1186/1472-6831-9-13.

16. Shetty SR, Munshi AK. Oral habits in children--a prevalence study. J Indian Soc Pedod Prev Dent. 1998 Jun;16(2):61-6.

17. Quashie-Williams R, Costa OO, Isiekwe MC. Oral habits, prevalence and effects on occlusion of 4-15 year old school children in Lagos, Nigeria. Niger Postgrad Med J. 2010 Jun;17(2):113-7.
18. Leite-Cavalcanti A, Medeiros-Bezerra PK, Moura C. Breastfeeding, bottle-feeding, sucking habits and malocclusion in Brazilian preschool children. Rev Salud Publica (Bogota). 2007 Apr-Jun;9(2):194-204. Portuguese.

19. Hebling SR, Cortellazzi KL, Tagliaferro EP, Hebling E, Ambrosano GM, Meneghim MC, et al. Relationship between malocclusion and behavioral, demographic and socioeconomic variables: a cross-sectional study of 5 -year-olds. J Clin Pediatr Dent. 2008 Fall;33(1):75-9.

20. Thomaz EB, Cangussu MCT, Assis AMO. Malocclusion and deleterious oral habits among adolescents in a developing area in northeastern Brazil. Braz Oral Res. 2013 JanFeb;27(1):62-9.

21. Morley DS. Psychophysiological reactivity to stress in nail biters. Int J Neurosci. 2000 Jul-Aug;103(1-4):139-54.

22. Winocur E, Littner D, Adams I, Gavish A. Oral habits and their association with signs and symptoms of temporomandibular disorders in adolescents:a gender comparison. Oral Surg Oral Med Oral Pathol Oral Radiol Endod. 2006 Oct;102(4):482-7.

23. Sardenberg F, Martins MT, Bendo CB, Pordeus IA, Paiva SM, Auad SM, et al. Malocclusion and oral health-related quality of life in Brazilian schoolchildren. Angle Orthod. Epub 2012 May 21.

24. Hainsworth JM, Hill KB, Rice A, Fairbrother KJ. Psychosocial characteristics of adults who experience difficulties with retching. J Dent. 2008 Jul;36(7):494-9.

25. Peres KG, Barros AJD, Anselmi L, Peres MA, Barros FC. Does malocclusion influence the adolescent's satisfaction with appearance? A cross-sectional study nested in a Brazilian birth cohort. Community Dent Oral Epidemiol. 2008 Apr;36(2):137-43.

26. Tanaka OM, Vitral RWF, Tanaka GY, Guerrero AP, Camargo ES. Nailbiting, or onychophagia: a special habit. Am J Orthod Dentofacial Orthop. 2008 Aug;134(2):305-8.

27. Schroeder U, Santibanex G. Gagging as a symptom of induced anxiety reactions. Stomatol DDR. 1978 Aug;28(8):576-80. German.

28. Akarslan ZZ, Biçer AZY. Utility of the gagging problem assessment questionnaire in assessing patient sensitivity to dental treatments. J Oral Rehabil. 2012 Dec;39(12):948-55. 